Volume 1, Issue 1, 2011, 015-023

Received: 7.02.2011 / Accepted: 12.02.2011 / Published on-line: 15.02.2011

\title{
Antifungal activity of some vegetal extracts on Candida biofilms developed on inert substratum
}

\section{Crina Saviuc**1, Alexandru Mihai Grumezescu $^{2}$, Eliza Oprea ${ }^{3}$, Valeria Radulescu ${ }^{4}$, Luminita Dascalu $^{1}$, Mariana Carmen Chifiriuc ${ }^{1}$, Marcela Bucur ${ }^{1}$, Otilia Banu ${ }^{5}$, Veronica Lazar ${ }^{1}$}

\section{ABSTRACT}

Essential oils extracted from Citrus maxima (epicarp), Anethum graveolens (fruits) and Picea abies (leaves) were investigated for their ability to inhibit the growth and coordinated expression of virulence factors in Candida sp. strains recently isolated from clinical specimens, identified by using Vitek II automatic system and tested for susceptibility to antifungal drugs by E-test method. Plant materials were hydrodistiled in a Neo Clevenger type apparatus. Qualitative screening was performed using killing time curve and an adapted disk diffusion method. The microtiter method was used for establishing the influence of the essential oils on the microbial adherence to inert substrata. Subinhibitory concentrations of the tested essential oils were studied for their interference with the expression of soluble enzymatic virulence factors. All extracts exhibited an inhibitory activity of variable intensity on the microbial growth of planktonic as well as adherent strains, demonstrating the potential effect of the active compounds on the coordinated expression of virulence factors and consequently, on the initiation and progression of a fungal biofilm associated infection.

Keywords: Anethum graveolens, Citrus maxima, Picera abies, essential oils, Candida sp., antifungal activity, fungal biofilm

\section{Introduction}

In the last decades, fungi have emerged as a major causes of human disease, particularly among immunocompromised individuals and hospitalized patients with serious underlying conditions [1]. Fungi can affect various parts of the body, ranging from superficial and cutaneous to deeply invasive and disseminated infections, aggravated by biofilm development on vaginal and oral epithelia, as well as on dental prostheses and implanted devices. Biofilms represent a niche for microorganisms where they are protected from both the host immune system and antimicrobial therapies [2]. Biofilm formation is preceded by microbial cells adherence to tissues or medical

\footnotetext{
${ }^{1}$ University of Bucharest, Faculty of Biology, Microbiology University Department

*Coresponding author email address: crina.saviuc@yahoo.com

${ }^{2}$ University Politechnica of Bucharest, Faculty of Applied Chemistry and Materials Science, Organic Chemistry Department

${ }^{3}$ University of Bucharest, Faculty of Chemistry, Organic Chemistry Department

${ }^{4}$ Univeristy of Medicine and Pharmacy Carol Davila, Organic Chemistry Department

${ }^{5}$ Institute of Cardiovascular Diseases Prof. C.C. Iliescu, Bucharest
} 
devices, presumably resulting in a change in organism morphology and behavior, representing the most important virulence factor in invasive candidiasis pathogenesis, followed by secretion of a number of hydrolytic enzymes [1]. Treatment of fungal infections, particularly nosocomial ones, has lagged behind bacterial chemotherapy and there are substantially fewer antifungal than antibacterial drugs due to the toxic side effects (fungi being eukaryotic cells similar in structure with the host cells) and limited solutions for the development of new classes of antibiotics [3]. Therefore, the use of essential oils for the prevention and treatment of fungal infection has been gaining popularity within the research field over the past decade, using in vitro and in vivo models, even clinical studies, the anti-infective properties of essential oils referring to their microbicidal or anti-pathogenic effect (exhibited by influencing the coordinated expression of virulence factors in opportunistic pathogens) $[1,4,5,6]$. It seems that essential oils have a stronger antimicrobial effect on biofilm grown cells as compared with that other vegetal compound, due to their better diffusion rates and easy contact with the target structures, facilitated by the saturation of incubation atmosphere with their low density fraction [5]. In this context the aim of our study was to evaluate the inhibitory activity of essential oils extracted from Anethum graveolens, Citrus maxima and Picea abies on planktonic and adhered fungal clinical strains.

\section{Experimental section}

2.1. Fungal strains. We used 6 strains, recently isolated from clinical specimens, belonging to Candida and Saccharomyces genera. They were identified by Vitek II automatic system, and tested for susceptibility to currently used antifungal drugs by E-test (voriconazole, itraconazole, caspofungin B, fluconazole, flucytosin, amphotericin B). The identified strains and their isolation sources are presented in table 1.

Tabel 1. Clinical origin and species assignment of the fungal strains

\begin{tabular}{l|l}
\hline \multicolumn{1}{c|}{ Fungal strains } & \multicolumn{1}{c}{ Clinical specimen } \\
\hline Candida albicans 2026 & Bronchotracheal secretions \\
\hline Candida tropicalis 4694 & Bronchotracheal secretions \\
\hline Candida famata 198 & sputum \\
\hline Candida glabrata 1957 & Bronchotracheal secretions \\
\hline Candida krusei Y5 & Vaginal swab \\
\hline Saccharomyces cerevisiae 200 & Sputum \\
\hline
\end{tabular}

2.2. Essential oils. Dried samples from different vegetative and reproduction organs of selected plants - A. graveolens (fruits), C. maxima (epicarp), P. abies (leaves) - were hydrodistilled in a Neo Clevenger type apparatus performing simple and microwave assisted hydrodistilation. Chemical composition was settled by GC-MS analysis. Stock solutions 1:1 (v/v) with dimethylsulfoxide (DMSO) were prepared for bioassays.

2.3. Qualitative screening of the antimicrobial activity. We used microbial suspensions of 1$1.5 \times 10^{8} \mathrm{CFU} / \mathrm{mL}$ or $0.5 \mathrm{McF}$ arland density obtained from $15-18 \mathrm{~h}$ microbial cultures grown on solid medium (Sabouraud agar). The qualitative screening of the susceptibility spectra of different fungal strains to the essential oils was performed by killing time curve assay and an adapted diffusion 
method. For the killing time curve the microbial strains were kept in contact with the essential oil for 1', 3', 5,' 15' and 30', viable cell counts being thereafter performed in order to appreciate the microbiostatic or microbicidal effect, as well as the persistence of the antimicrobial activity. Diffusion method was performed in three working variants: spot, well and disk diffusion technique.

2.4. Quantitative assay of the antimicrobial activity for establishing the minimal inhibitory concentration (MIC). Serial twofold (starting from 250 to $0.05 \mu \mathrm{L} / \mathrm{mL}$ ) and tenfold (for $A$. graveolens starting from 50 to $5 \times 10^{-5} \mu \mathrm{L} / \mathrm{mL}$ ) dilutions of the essential oil stock solutions were performed in glucose broth, distributed in 96 multiwell plates, and further inoculated with a standard inoculum of microbial strains. The plates were coated with parafilm tape for sealing, incubated at $30^{\circ} \mathrm{C}$ for $24 \mathrm{~h}$ and then tested for cell growth. The lowest concentration of extract that completely inhibited cell growth was considered as MIC value, assessed by reading the $620 \mathrm{~nm}$ absorbance (A620) [4].

\subsection{Influence on coordinated expression of virulence factors in Candida strains.}

2.5.1. Filamentation assay: Microbial suspensions of 2 McFarland density were mixed 1:1 v/v with bovine serum, incubated for $2 \mathrm{~h}$ at $37^{\circ} \mathrm{C}$, and examined with the objective 40x.

2.5.2. Adherence to the inert substrata. A simple microtiter method was performed for biomass appreciation. After following the MIC protocol fungal biofilms developed after $24 \mathrm{~h}$ on plastic wells, were fixed by cold methanol for 5 min, stained by violet crystal, suspended by $33 \%$ acetic acid and quantified by measuring the A490 nm [7].

2.5.3. Soluble enzymatic virulence factors. The microbial strains were cultivated in glucose broth with and without addition of subinhibitory concentrations of tested essential oils stock solutions and subsequently. The obtained overnight microbial cultures were spotted onto special media for assessing the following virulence factors production [8]. Plate haemolysis: the strains were streaked on blood Sabouraud agar plates containing 5\% (vol/vol) sheep blood in order to obtain isolated colonies. After incubation at $37^{\circ} \mathrm{C}$ for $24 \mathrm{~h}$ the clear zone (total lysis of red blood cells) around the colonies was registered as positive reaction. Protease activity: it was determined by using 3\% gelatine agar as substrate medium (Cantacuzino Institute Media Laboratory). After incubation at $37^{\circ} \mathrm{C}$ up to $48 \mathrm{~h}$, a clear zone surrounding the growth area indicated gelatine proteolysis (gelatinase presence). DNA-se production: it was studied using DNA agar medium. The strains were spotted and after incubation at $37^{\circ} \mathrm{C}$ up to $48 \mathrm{~h}$, clear pink zone around the colonies was registered as positive reaction. Lipase production: the cultures were spotted on Tween 80 agar with a substrate at a final concentration of $1 \%$ and were incubated at $37 \mathrm{oC}$ up to 7 days. An opaque (precipitation) zone around the spot was registered as positive reaction. Esculin hydrolysis: The esculin hydrolysis was evidenced onto Esculin containing agar medium. In the presence of an iron salt, esculethol (an iron chelating agents) forms a brown-black complex that diffuses into the surrounding medium interpreted as positive reaction. Amylase production: was studied on a starch Sabouraud agar medium and quantified as a colorless zone around the culture contrasting with the blue color resulted after adding Lugol solution.

\section{Results section}

3.1. Essential oils. Obtaining and physico-chemical characterization of essential oils were subject of previously works $[9,10,11]$. The average yields of essential oil $(\mathrm{v} / \mathrm{w} \%$, normalized to the part of the plant mass dried weight) extracted from A. graveolens, C. maxima and P. abies were $3.77 \%, 0.87 \%$ 
and $1.01 \%$ respectively. In the complex matrix of selected essential oils a variety of classes of organic compounds known as antimicrobial agents were identified: mono- and sesquiterpenes and related terpenoids - esthers, ethers or carbonilic derivates (tables 2).

Tabel 1. The main classes of chemical compounds percentage

\begin{tabular}{llll}
\hline & P. abies & C. maxima & A. graveolens \\
\hline monoterpens & $25.11 \%$ & $97.37 \%$ & $57.64 \%$ \\
\hline
\end{tabular}

sesquiterpenes $\quad 16.76 \%$

\begin{tabular}{llll}
\hline alkanes & - & $0.31 \%$ & - \\
\hline terpenoids & $61.5 \%$ & $0,12 \%$ & $39.56 \%$ \\
\hline
\end{tabular}

The $C$. maxima and A. graveolens essential oils are rich in monoterpens, while $P$. abies is rich in oxygenated compounds. The main compounds for each essential oil are presented in tables 3, 4 and 5.

Table 2. The main compounds and their percentage in Picea abies essential oil

Limonene Camphene Bornyl acetate

Table 4. The main compounds and their percentage in $A$. graveolens essential oil

$\alpha$-phellandrene carvone limonene

Table 5. The main compounds and their percentage in C. maxima essential oil

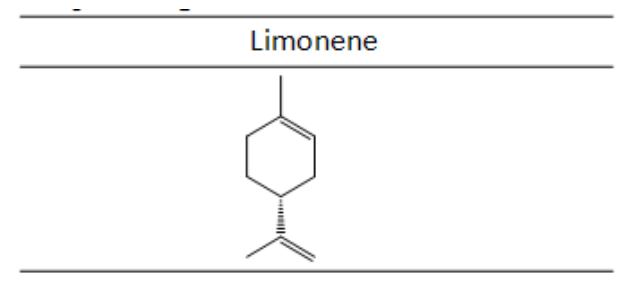

$88.87 \%$

3.3. Qualitative assays. Since essential oils are subject for pharmacological studies, tests on their antimicrobial activities have been done frequently [4]. Fractions from essential oils were assessed for antimicrobial activity to, i.e. thymol, geraniol, citral and linalool being 20, respectively 7,1; 5, 2 and 5 times stronger then phenol. Some volatile compounds from tested essential oils matrix were related with the antimicrobial activity: $\alpha$ - and $\beta$-pinene [12], $\delta$-3-carene [12], carvone [13] p-cymene [14], ocimene [15], limonene [15, 16], $\gamma$-terpinene [14], camphene [4], 1.8-cineol [4], linalool [17], bornyl acetate [14], and nerolidol [18]. All tested essential oil exhibited antimicrobial activity on the tested strains. The two qualitative assays highlighted the antimicrobial activity from different points of view. The diffusion technique shows the antifungal effect tested for $P$. abies essential oil, while the killing time curve revealed the early microbicidal and vapor phase effect for $A$. graveoens and $C$. maxima, manifested sometimes even to positive controls spotted inocula (in case of $C$. glabrata, $C$. tropicalis) (Figure 1). 


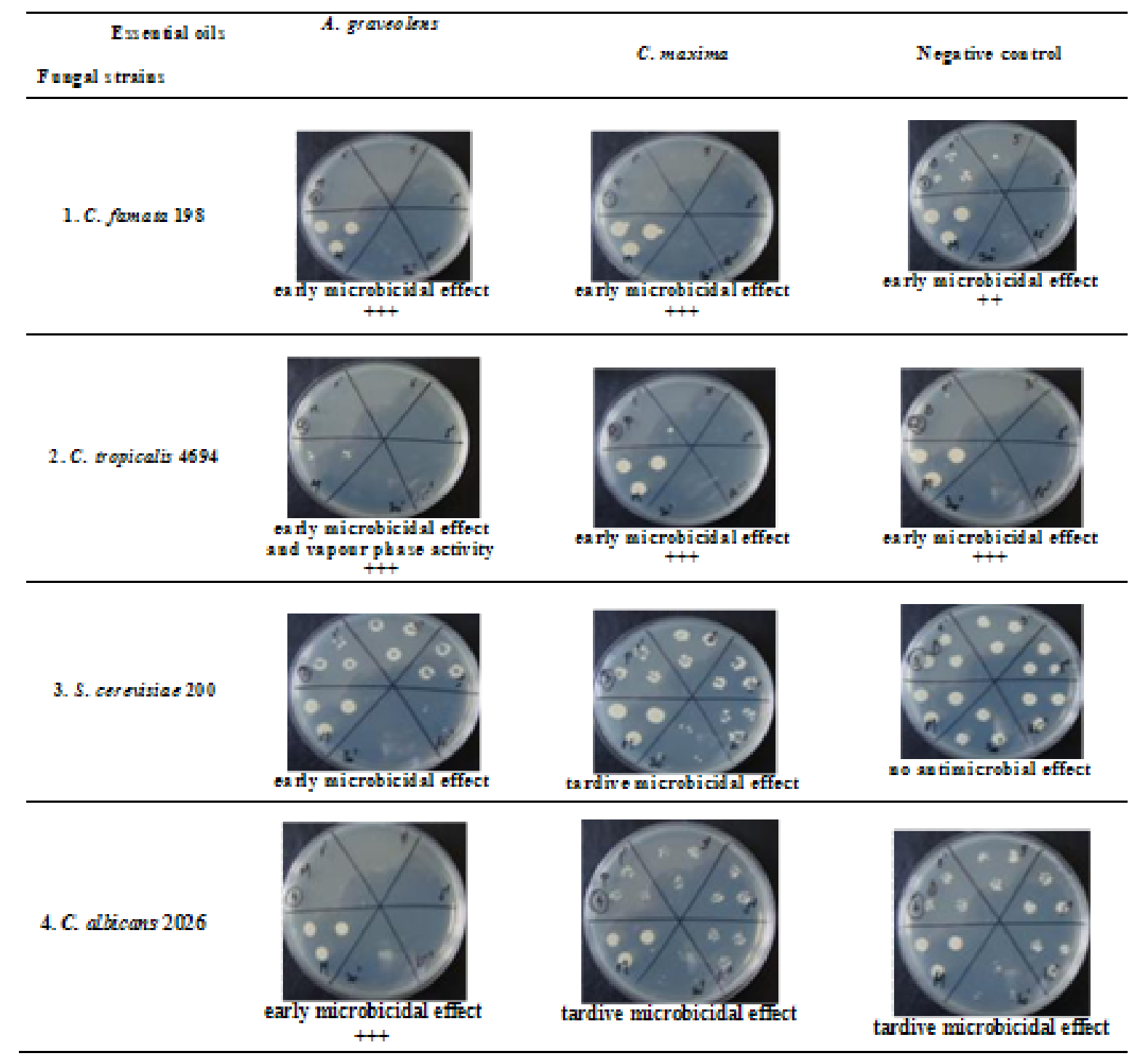

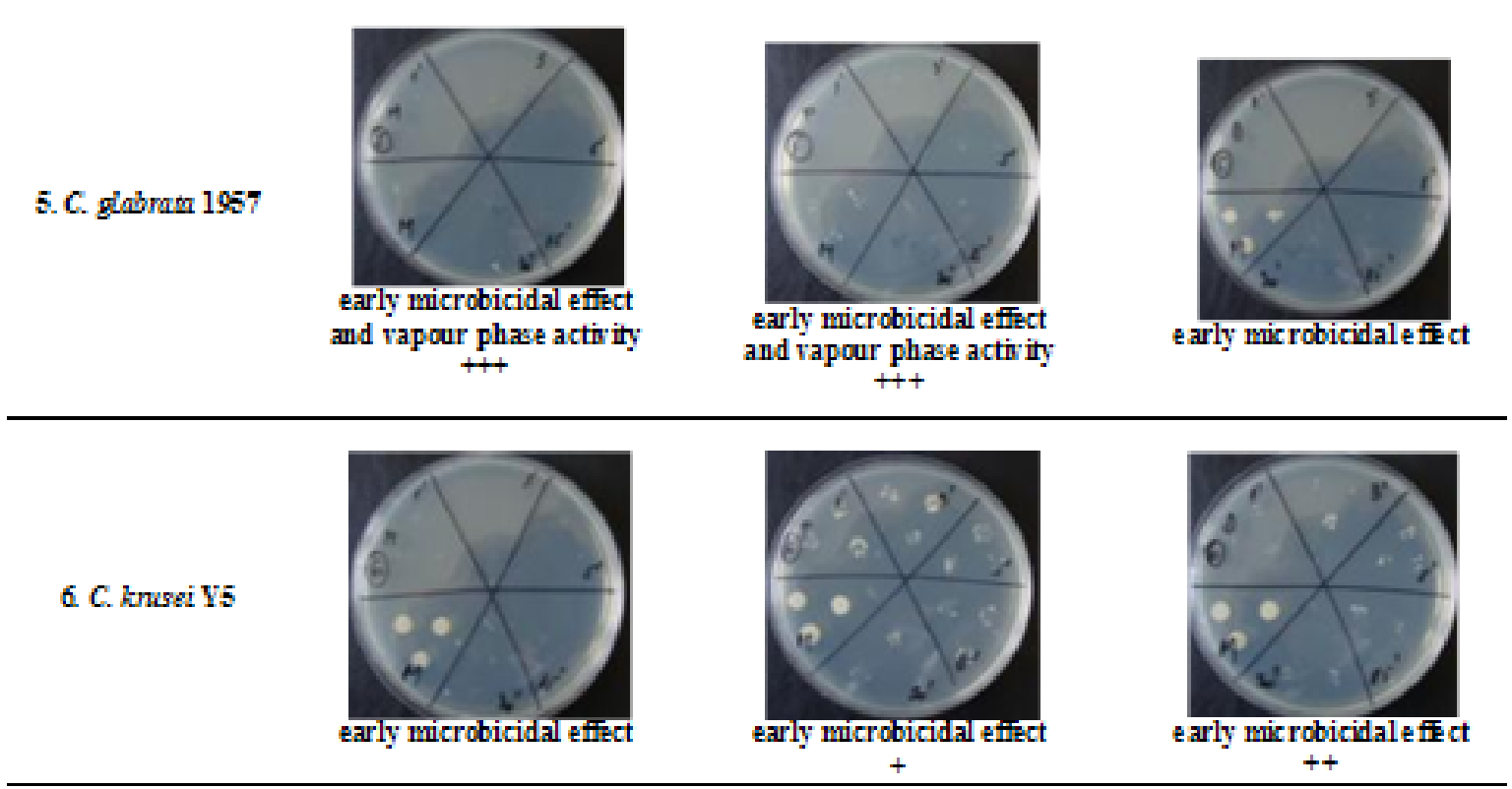

Figure 1. The aspect of colonies grown on solid media after spotting ten-fold dilutions of liquid cultures developed in the presence of essential oils 


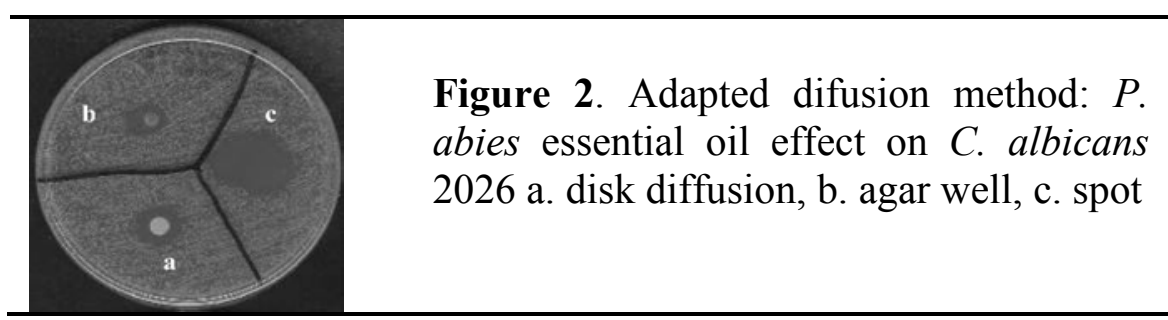

The advantage of killing time method results is establishing the early or late microbicidal effect character which can be correlated with the mechanism of antimicrobial activity. In this respect, the major effect of tested essential oils was exhibited at the level of the cellular wall. Our results are in accordance with other literature data, mentioning that yeasts turned out to be susceptible toward essential oil vapors; possibly, in yeast, the biosynthesis of chitin being inhibited by plant volatile compounds [4].

3.4. MIC values. The MIC values for essential oils are subject of a great variability [4], thus the qualitative assays should be the starting points in the identification of the active compounds (Figure 3). However, the MIC values for the tested essential oils, ranged from 0.05 to $6.95 \mu \mathrm{L} / \mathrm{mL}, A$. graveolens exhibiting inhibitory activity at the lowest MIC values against all tested strains.

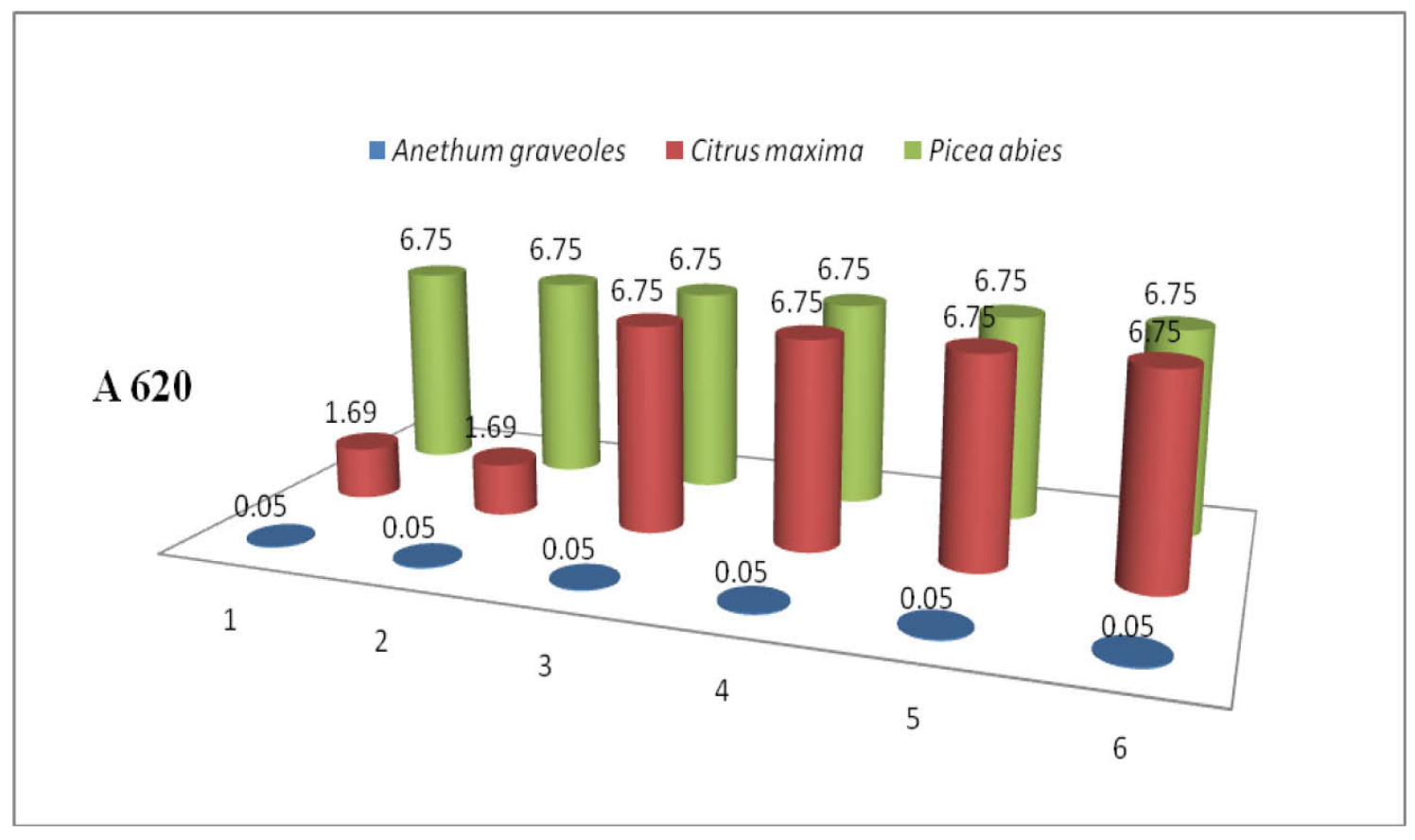

Figure 3. MIC of essential oils on the tested strains

\subsection{Influence on virulence factors expression}

3.5.1. Adherence to the inert substrata. The essential oils inhibited the microbial adherence on inert substrata at subinhibitory concentrations (Figure 4).

3.5.2. Soluble enzymatic virulence factors. All tested enzymes were influenced by subinhibitory concentrations of the tested essential oils, except for the heamolysins. DNA-se production was inhibited for three strains in the presence of various tested essential oils, while lipases were suppressed in all strains. Essential oil from C. maxima peels was the most active in inhibiting soluble enzymatic virulence factors production, in case of DNA-se (for S. cerevisiae 200 and C. albicans 
2026), lipase (for C. glabrata 1957) amylase (for C. albicans 2026) and esculin hydrolysis (for $C$. tropicalis 4694).
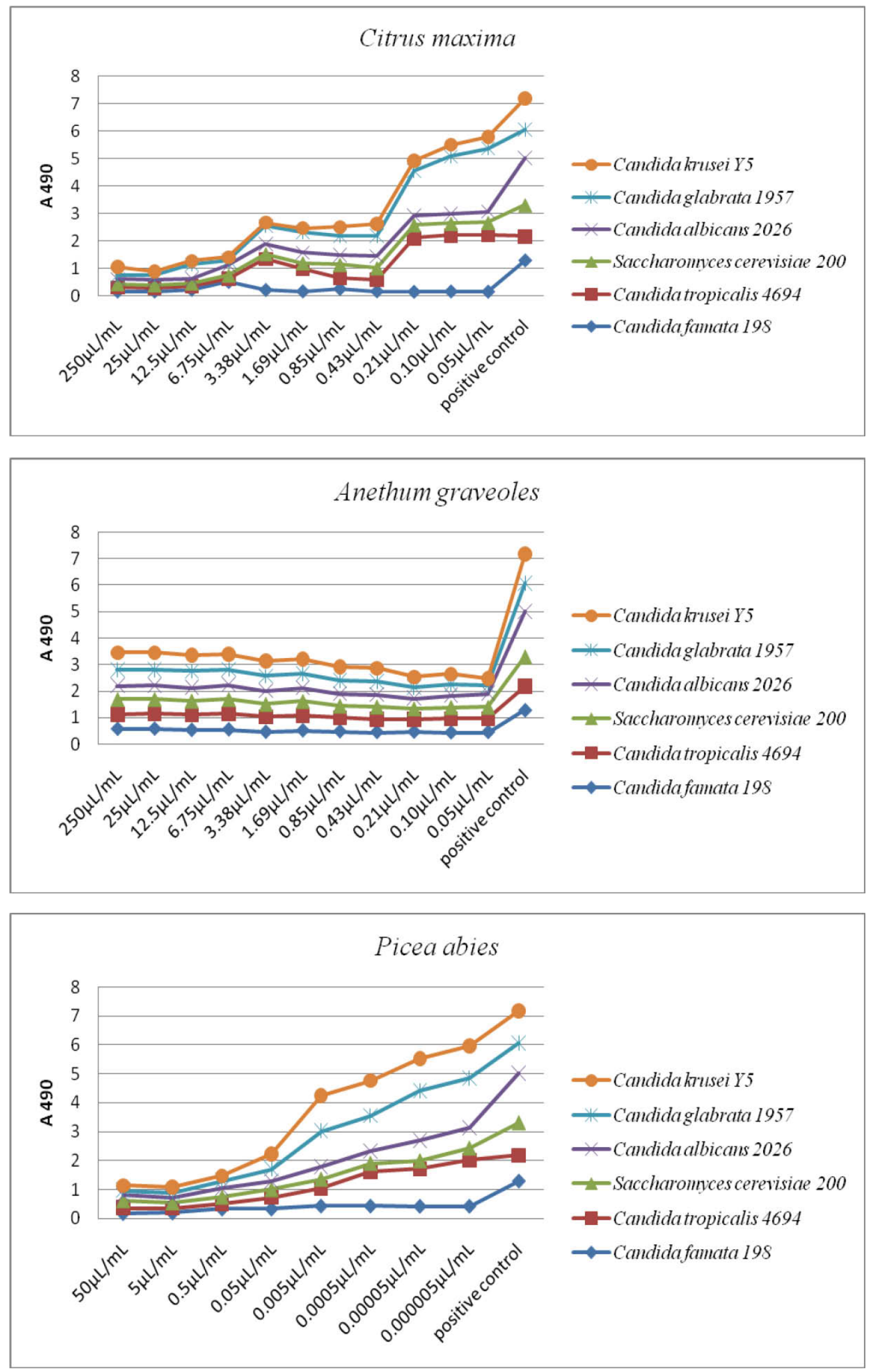

Figure 4. The effect of different concentrations of essential oils on the fungal biofilms developed on plastic sbstrate 
Table 6. Influence of essential oils on the expression of soluble enzymatic virulence factors

\begin{tabular}{|c|c|c|c|c|c|}
\hline Vir. factors & Fungal strains (code) & $\begin{array}{c}\text { A. } \\
\text { graveoles }\end{array}$ & $\begin{array}{c}P . \\
\text { abies }\end{array}$ & $\begin{array}{c}C . \\
\text { maxima }\end{array}$ & $\begin{array}{c}\text { Untreated } \\
\text { Control }\end{array}$ \\
\hline \multirow{6}{*}{ DNA-se } & C. famata 198 (1) & + & - & - & - \\
\hline & C. tropicalis 4694 (2) & + & - & - & - \\
\hline & S. cerevisiae $200(3)$ & - & + & - & + \\
\hline & C. albicans 2026 (4) & - & - & - & + \\
\hline & C. glabrata 1957 (5) & + & - & + & + \\
\hline & C. krusei Y5 (6) & + & - & - & - \\
\hline \multirow{6}{*}{ Lipase activity } & 1 & - & - & + & - \\
\hline & 2 & - & + & - & + \\
\hline & 3 & - & - & + & - \\
\hline & 4 & - & - & + & - \\
\hline & 5 & - & + & - & + \\
\hline & 6 & - & - & + & - \\
\hline \multirow{6}{*}{$\begin{array}{l}\text { Esculin } \\
\text { hydrolysis }\end{array}$} & 1 & - & - & - & - \\
\hline & 2 & + & + & - & + \\
\hline & 3 & - & - & - & - \\
\hline & 4 & - & - & - & - \\
\hline & 5 & + & + & + & + \\
\hline & 6 & + & - & - & - \\
\hline \multirow{6}{*}{$\begin{array}{l}\text { Amylase } \\
\text { production }\end{array}$} & 1 & - & - & $\begin{array}{l}\text { growing } \\
\text { absence }\end{array}$ & - \\
\hline & 2 & - & - & - & - \\
\hline & 3 & - & - & - & - \\
\hline & 4 & + & + & - & + \\
\hline & 5 & - & - & - & - \\
\hline & 6 & - & - & - & - \\
\hline
\end{tabular}

\section{Conclusions}

The essential oils possess antimicrobial activity of different intensity depending on their chemical composition and the tested strain. A. graveolens essential oil exhibited superior antimicrobial activity probably due to his balanced composition in terpenes and terpenoides. The most evident inhibitory effect was noticed for strains C. tropicalis 4694 and C. glabrata 1957 treated with $A$. graveolens essential oil. The obtained results recommend the use of these plant extracts for the development of new strategies for the control of biofilm related fungal infections.

\section{References}

[1] Trofa D., Gácser A., Nosanchuk JD., Candida parapsilosis, an emerging fungal pathogen, Clin Microbiol Rev., 21, 4, 606-625, 2008 
[2] Andes D., Nett J., Oschel P., Albrecht R., Marchillo K., Pitula A., Development and Characterization of an In Vivo Central Venous Catheter Candida albicans Biofilm Model, Infection and Immunity, 72, 10, 60236031, 2004

[3] Jiang Z., Kullberg B.J., Van der Lee H., Vasil A., Hale J., Mant, Hancock C. R., Vasil M., Netea M., Hodges R., Effects of hydrophobicity on the antifungal activity of $\alpha$-helical antimicrobial peptides, Chem Biol Drug Des.,72, 6, 483-495, 2008

[4] Hüsnü Can Bașer K., Buchbauer G.,"Handbook of essential oils. Science, Technology and Applications”, CRC Press, London, 2010

[5] Tyagi A.K., Malik A., Liquid and vapour-phase antifungal activities of selected essential oils against Candida albicans: microscopic observations and chemical characterization of cymbopogon citrates, BMC Complementary and Alternative Medicine, 10, 65, 2010

[6] Prakash B., Shukla R., Singh P., Mishra P. K., Dubey N. K., Kharwar R. N., Efficacy of chemically characterized Ocimum gratissimum L. essential oil as an antioxidant and a safe plant based antimicrobial against fungal and aflatoxin B1contamination of spices, Food Research International, 44, 385-390, 2011

[7] Lazãr V., Chifiriuc M.C., Architecture and physiology of Microbial Biofilms, Roum. Arch. Microbiol. Immunol. 69, 3, 92-98, 2010

[8] Lazar V., Balotescu M.C., Moldovan L., Vasilescu G., Petrache L.M., Bulai D., Cernat R., Comparative evaluation of qualitative and quantitative methods used in the study of antifungal and antibacterial activity of hydroalcoholic vegetal extracts, Roum. Biotechnol. Lett., 10, 2225-2232, 2005

[9] Saviuc C., Oprea E., Rădulescu V., Ilieş D., Chifiriuc C., Măruțescu L., Popa M., Bleotu C., Lazăr V., Chemical and biological studies of Anethum graveolens essential oil, 7th International Conference of Chemical Societies from South-East European Countries on "Chemistry - Beauty and Application", Bucharest, 15-17 September, 2010

[10] Saviuc C., Dascãlu L., Chifiriuc M.C., Rãdulescu V., Oprea E., Popa M., Hristu R., Stanciu G., Lazãr V., The inhibitory activity of Pomelo essential oil on the bacterial biofilms development on soft contact lenses, Romanian Archives of Microbiology and Immunology, 69, 3, 140-145, 2010

[11] Rădulescu V., Saviuc C., Chifiriuc C., Oprea E., Ilieş D.C., Măruțescu L., Lazăr V., Chemical compozition and antimicrobial activity of essential oil from shoots spruce (Picea abies L.), Rev. Chem. (Bucharest), 62, 1, 69-74, 2011

[12] Gershenzon J., Dudareva N., The function of terpene natural products in the natural world, Nat Chem Biol, 3, 7, 408, 2007

[13] Carla C.C.R. de Carvalho, M. Manuela R. da Fonseca, Carvone: Why and how should one bother to produce this terpene, Food Chemistry, 95, 413-422, 2006

[14] Burt S., Essential oils: their antibacterial properties and potential applications in foods-a review, Int. J. Food Microbiol., 94, 3, 223, 2004

[15] Deans S.G., Mint. The Genus Mentha, Edited by Brain. M. Lawrence, CRC Press, 2007

[16] Aggarwal K.K., Khanuja S.P.S., Ahmad A., Kumar T.R.S., Gupta V. K., Kumar S., Antimicrobial activity profiles of the two enatiomers of limonene and carvone isolated from the oils of Mentha spicata and Anethum sowa, Flavour. Frag. J., 17, 1, 59, 2002

[17] Delaquis P.J., Stanich K., Girard B., Mazza G., Antimicrobial activity of individual and mixed fractions of dill, cilantro, coriander and Eucalyptus essential oils, Int. J. Food Microbiol., 74, 1, 101, 2002

[18] Hada T., Shiraishi A., Furuse S., Inoue Y., Hamashima H., Masuda K., Shiojima K., Shimada J., Inhibitory effects of terpenes on the growth of Staphylococcus aureus, Nat. Med., 57, 2, 64, 2003

Acknowledgement: This research was financed by the European Funding Program POSDRU 107/1.5/S/80765 